\title{
Dynamic Compensation of Ultra-Low-Range Pressure Sensors
}

\author{
Paul Nathan, Rebecca Manning, and David M. Birch
}

\begin{abstract}
A technique has been developed to compensate pressure readings from arrays of highly sensitive membrane-type pressure sensors for deflections caused by acceleration normal to the plane of the membrane using a single inertial measurement unit. By normalizing the fourth-order unsteady Kirchoff-Love equation, it can be shown that inertial body pseudoforces and applied surface pressure elicit a similar and additive response from the sensors. Inertial effects arising from linear and angular acceleration as well as angular velocity may therefore be converted to 'pseudopressures' and eliminated by means of a simple linear compensation process which can be calibrated using only gravity. To demonstrate, signals from a conventional six-axis inertial measurement unit (including three orthogonal components each of angular velocity and linear acceleration) are used to provide an approximation of the acceleration of the sensing dies within a seven-channel distributed array of ultralow pressure sensors. Applying the proposed correction reduces the maximum full-scale uncertainty of the measurements by as much as $\mathbf{5 0 \%}$.
\end{abstract}

\section{INTRODUCTION}

$\mathbf{I}$ MPROVEMENTS in micro-manufacturing capability and precision have resulted now in the wide availability of ultralow-range membrane-type pressure sensors; some have fullscale ranges as low as $160 \mathrm{~Pa}(1)$. When pressure sensors are used in flow measurement systems (such as Pitot-static probes and head-loss sensing flow meters), the nonlienar relationship between stagnation pressure and velocity significantly amplifies uncertainties at low speeds (2). For the case of directional velocity probes in particular, even smaller pressure differences must typically be resolved, and so sensor uncertainty can dominate $(3 ; 4)$. Pressure sensors with ultra-low full-scale ranges (and therefore uncertainties) are consequently in high demand for applications in low-speed aerodynamics.

Typical membrane-type pressure sensors consist of a thin semiconductor membrane (usually ion-beam etched from a silicon substrate) patterned with piezoresistive strain-gauges. Changes in the applied pressure elastically deform the membrane, resulting in a measurable surface strain. The sensors are usually calibrated for both applied pressure and temperature, and the temperature sensitivity is then eliminated in postprocessing. The membrane, however, has nonzero mass and is therefore also sensitive also to acceleration in a direction normal to its plane $(5 ; 6)$.

To compensate for acceleration-sensitivity, one approach has been to etch two identical sensing membranes into the same

P. Nathan and R. Manning are with Surrey Sensors Ltd., Unit 13 Parklands, Railton Road, Guildford, Surrey, GU2 9JX, UK.

D. M. Birch is with the University of Surrey, Department of Mechanical Engineering Sciences, Guildford, Surrey, GU2 7XH, UK; email:d.birch@surrey.ac.uk. die and pattern half a Wheatstone bridge onto each, but on opposite faces of the die. Only one membrane is exposed to the applied pressure, but both are equally exposed to the inertial loads: the inertial effects will in this way be balanced out (7). This approach, however, necessarily requires $(a)$ that the membranes are identical (so that both will experience the same strains in response to an applied inertial load), and (b) that the membranes are essentially co-located (so that in six degree-of-freedom motion the membranes experience the same accelerations). For the case of ultra-low range pressure sensors, the die membranes can be as little as $10 \mu \mathrm{m}$ in thickness, and the unit-to-unit variability is proportionally much higher than higher-range sensors (1). Also, since most ultra-low range sensor products are already housed in large $(\sim 1 \mathrm{~cm})$ enclosures, co-location is not physically possible. For the case of arrays of ultra-low range pressure sensors, then, this direct balancing approach is not practical and some form of post-processing correction is necessary.

\section{A. Pressure measurement error due to inertial effects}

The error in pressure measurements arising from the acceleration of the membrane's inertial frame can be predicted from dimensional analysis alone, such that

$$
\varepsilon_{P} \sim \rho h a_{z}
$$

where $\rho$ is the density of the membrane material, $h$ is the membrane thickness, and $a_{z}$ is the component of acceleration normal to the plane. For a typical ultra-low range siliconbased sensor die, $\rho \sim 2330 \mathrm{~kg} / \mathrm{m}^{3}$ and $h \sim 20 \mu \mathrm{m}$, yielding an approximate response to acceleration of $0.047 \mathrm{~Pa} /\left(\mathrm{m} / \mathrm{s}^{2}\right)$. With this sensitivity, gravity alone can result in a full-scale error of approximately $\pm 0.3 \%$ for a typical very-low range sensor, making changes in orientation the largest potential source of error after drift and residual temperature sensitivity (1). Indeed, the configuration of high-sensitivity membranetype pressure sensors is not altogether dissimilar from that of advanced monolithic micro-electromechanical linear accelerometers themselves (8).

Inertial measurement units (IMUs) currently available are able to provide three orthogonal components of both linear acceleration and angular velocity. Because these IMUs are also small and inexpensive, they can be easily added to arrays of surface-mounted pressure sensors. The IMU's signals may then be used to resolve the linear acceleration vector at any known location within its own inertial frame, when subjected to arbitrary translational and rotational motion. With the local acceleration vector, it should then be possible to compensate 
measurements from a pressure sensor anywhere within the inertial frame for the 'pseudopressure' resulting from any arbitrary acceleration.

\section{B. Pressure sensor response model}

Before it is possible to propose any correction for acceleration, it is first necessary to establish a dynamic response model for a typical membrane-type pressure sensor.

Consider a typical pressure sensing die, consisting of a membrane of uniform thickness and density, having rigidly fixed boundary conditions. We will define an inertial reference frame $p$ fixed to the membrane boundary condition such that the $x-y$ plane coincides with the plane of the membrane. The inertial frame undergoes an arbitrary acceleration, while the membrane is simultaneously subjected to a pressure difference.

Next, we assume that the material is linear and elastic, and that the material deformations are small. These are normal design requirements for pressure dies: otherwise, the die would have unacceptably high hysteresis or low cycle life. Then, the in-plane deformation of the membrane (and therefore any effect of in-plane acceleration) is negligible and the out-ofplane deflection $w(x, y ; t)$ of the membrane is given by the Kirchoff-Love equation,

$$
\frac{\partial^{4} w}{\partial x^{4}}+2 \frac{\partial^{4} w}{\partial x^{2} \partial y^{2}}+\frac{\partial^{4} w}{\partial y^{4}}=P^{*}-\left(\frac{\partial^{2} w}{\partial \tau^{2}}+\frac{\partial^{2} w^{\prime}}{\partial \tau^{2}}\right)
$$

where $x$ and $y$ represent locations on the membrane normalized against some characteristic length scale $L$ (which can be taken, for example, as the side-length of the membrane); $P^{*}=P L^{3} / D$ is a dimensionless pressure normalized against the material bending stiffness $D=h^{3} E /\left(12-12 v^{2}\right) ; E$ and $v$ are the Young's modulus and Poisson ratio of the membrane material, respectively; $w^{\prime}(t)$ is the position of the inertial frame $p$ along the $z$-axis relative to a fixed observer, and

$$
\tau=\frac{t}{L^{2}} \sqrt{\frac{D}{\rho h}}
$$

is a nondimensional time. This model may be again simplified if it is further assumed that the effects of membrane dynamics are negligible: this is common practice, as the modal frequencies of pressure sensors are usually orders of magnitude higher than their own measurement bandwidths (again, by design). This is equivalent to the approximation $\partial^{2} w^{\prime} / \partial \tau^{2} \gg \partial^{2} w / \partial \tau^{2}$.

In normal steady-state operation (in a fixed frame), a pressure sensor must have a monotonic response to applied pressure. Since the pressure is inferred from the strain field in the membrane surface, the left-hand side of eq. (2) must also be some monotonic function of pressure $s\left(P^{*}\right)$. Under these assumptions, equation (2) may be simply recast as

$$
s\left(P^{*}\right)=P^{*}-a_{P z}^{\prime *}
$$

where the substitution $a_{P z}^{\prime *}=\partial^{2} w^{\prime} / \partial \tau^{2}$ has been made for simplicity. Note that for the steady-state case, $w^{\prime}=0$ and $s\left(P^{*}\right)=P^{*}$.

Equation (4) demonstrates that, under the assumptions listed, the normalized deformation response of a membranetype pressure sensor will be the simple additive combination of the nondimensional pressure and nondimensional acceleration of the reference frame. It may also be noted here that the calibration of the pressure sensor can be included in the function $s$ without further approximation. Correcting calibrated pressure signals for the acceleration of the reference frame, then, reduces to obtaining values of $a_{P z}^{\prime *}$.

\section{Modelling inertial forces}

Consider now a typical board-mounted pressure sensor located on the same printed circuit board (PCB) as an IMU. The sensing membrane is assumed to be located somewhere within the sensor enclosure, although its exact position and orientation may not be precisely known. If the origin $o^{\prime}$ of an inertial reference frame is placed coincident with the origin of the IMU and fixed relative to the IMU, the position vector of the membrane in this frame may be expressed (in Cartesian coordinates) as $\mathbf{r}_{\mathbf{P} / \mathbf{o}^{\prime}}=x_{P} \mathbf{e}_{\mathbf{x}}+y_{P} \mathbf{e}_{\mathbf{y}}+z_{P} \mathbf{e}_{\mathbf{z}}$, where $\mathbf{e}_{\mathbf{i}}$ is a unit vector along the $i$ axis (see Figure 1). Since the position and orientation of the sensing membrane are fixed relative to $o^{\prime}$ (assuming that the PCB is rigid), the dimensional acceleration ap of the centre of the membrane relative to a fixed observer may be expressed as

$$
\mathrm{a}_{\mathbf{P}}=\mathrm{a}_{\mathrm{o}^{\prime}}+\boldsymbol{\alpha} \times \mathrm{r}_{\mathbf{P} / \mathrm{o}^{\prime}}+\boldsymbol{\omega} \times \boldsymbol{\omega} \times \mathrm{r}_{\mathrm{p} / \mathrm{o}^{\prime}}
$$

where $\mathbf{a}_{\mathbf{o}^{\prime}}$ is the linear acceleration vector of $o^{\prime}$ relative to a fixed observer, and $\boldsymbol{\omega}$ and $\boldsymbol{\alpha}$ are the angular velocity and acceleration vectors, respectively, of the $o^{\prime}$ system. Considering only $a_{P z}^{\prime}$ (the component of acceleration normal to the $x-y$ plane),

$$
\begin{aligned}
a_{P z}^{\prime} & =a_{o^{\prime} z}+\left(\omega_{x} \omega_{z}-\alpha_{y}\right) x_{P} \\
& +\left(\omega_{y} \omega_{z}+\alpha_{x}\right) y_{P}-\left(\omega_{x}^{2}+\omega_{y}^{2}\right) z_{P}
\end{aligned}
$$

where $a_{o^{\prime} z}$ is the $z$-component of the linear acceleration of the IMU, and the cartesian components of the angular velocity and angular acceleration vectors are defined such that $\boldsymbol{\omega}=$ $\omega_{x} \mathbf{e}_{\mathbf{x}}+\omega_{y} \mathbf{e}_{\mathbf{y}}+\omega_{z} \mathbf{e}_{\mathbf{z}}$ and $\boldsymbol{\alpha}=\alpha_{x} \mathbf{e}_{\mathbf{x}}+\alpha_{y} \mathbf{e}_{\mathbf{y}}+\alpha_{z} \mathbf{e}_{\mathbf{z}}$. Although the angular accelerations are not directly available from the IMU, they can be obtained by straightforward numerical differentiation of the angular velocities. The acceleration in eq. (6) may then be normalized as

$$
a_{P z}^{\prime *}=a_{P z}^{\prime} \frac{L^{3} \rho h}{D}
$$

For a board-mounted pressure sensor undergoing an arbitrary acceleration, then, the true normalized pressure $P^{*}$ may be obtained directly by substituting eq. (7) into eq. (4) and recognizing that the left-hand size of (4) is simply the (statically) calibrated output signal of the sensor, normalized as required.

\section{Limitations of corrections using linear accelerations only}

It is important to note here that equation (6) also provides a useful criterion for establishing whether an IMU with integrated gyroscopes (for measuring $\boldsymbol{\omega}$, and indirectly $\boldsymbol{\alpha}$ ) is necessarily required. This is especially important, as some of the existing correction techniques discussed depend on the use 


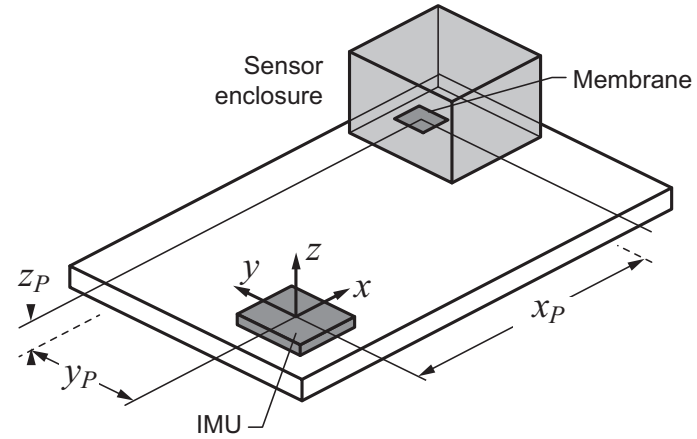

Fig. 1. Schematic of component placement, showing coordinate reference frame.

of a co-located 'dummy' membrane exposed to inertial loads only.

Consider two pressure sensing membranes mounted on the same PCB such that their planes are normal to the $z$-axis (as illustrated in figure 1). For simplicity, assume that the membranes have the same orientation, and both lie along the $x$-axis of the coordinate system, separated by some distance $\Delta x_{P}$. If mounted such that the height $z_{P}$ of the membrane above the PCB is small, the response of the membranes to rotational motion will be dominated by the component about the $y$-axis.

Next, assume that the PCB is subjected to a rotational oscillation about the $y$-axis only, such that

$$
\begin{aligned}
\theta(t) & =\theta_{0} \sin (2 \pi f t) \\
a_{o^{\prime} z} & =0
\end{aligned}
$$

where $\theta(t)$ is the angular position of the PCB about the $y$ axis, $\theta_{0}$ and $f$ are the amplitude and frequency of oscillation, respectively, and $t$ is time. Then, from eq. (6), the acceleration experienced by each sensor is given by

$$
a_{P z}^{\prime}=4 \pi^{2} f^{2} \theta_{0}\left(\sin (2 \pi f t) x_{P}-\theta_{0} \cos ^{2}(2 \pi f t) z_{P}\right)
$$

The difference in the accelerations experienced by the two sensors $\Delta a_{P z}^{\prime}$ will then be given by

$$
\Delta a_{P z}^{\prime}=4 \pi^{2} f^{2} \theta_{0} \sin (2 \pi f t) \Delta x_{P}
$$

Note that the second term in eq. (9) vanishes, as the two membranes are assumed to be at the same distance $z_{P}$ from the PCB.

Gravity effects are already known to be of significant importance in the uncertainty of ultra-low range pressure sensors. Therefore, $\Delta a_{P z}^{\prime}<2 g$ may be set as a reasonable criterion for the effects of this rotational oscillation on the pressure measurement to be negligible: this is equivalent to requiring that the maximum uncertainty resulting from the inertial effects is less than the uncertainty resulting from operating the membranes upside-down. Substituting this criterion into eq. (10) yields

$$
\frac{f^{2} \Delta x_{P}}{g} \theta_{0}<\frac{1}{2 \pi^{2}}
$$

If this criterion fails, using linear acceleration alone to compensate for momentum effects will result in an unacceptable increase in uncertainty.

For sensors with higher full-scale ranges, the effect of gravity is no longer a significant contributor to the uncertainty. In this case, a critical limiting acceleration may alternatively be obtained from eq. (1).

It is important to highlight here that the inclusion of the additional rotation terms in eq. (6) will also increase the uncertainty $\varepsilon_{a}$ of the acceleration $a_{P z}^{\prime}$. Taking as a worstcase condition a statistically isotropic rotational motion, eq. (6) yields (to within a first-order approximation)

$$
\varepsilon_{a}=\varepsilon_{l}+\left(4 \omega^{2}+2 \alpha\right) \varepsilon_{x}+2 \omega X_{P} \varepsilon_{\omega}+X_{P} \varepsilon_{\alpha}
$$

where $\omega$ and $\alpha$ are the angular velocity and angular acceleration scales, respectively; $\varepsilon_{l}$ is the uncertainty in the linear acceleration, $\varepsilon_{\omega}, \varepsilon_{\alpha}$ and $\varepsilon_{x}$ are the uncertainties in $\alpha, \omega$ and the positions of the components, respectively; $X_{P}=x_{P}+y_{P}$ and it is assumed that $X_{P} \gg z_{P}$. The uncertainty introduced by the three additional terms in eq. (12) must not exceed the benefit of the correction, and it must be ensured that $\varepsilon_{a} \ll a_{P z}^{\prime}$.

\section{EXPERIMENTAL DEMONSTRATION}

\section{A. Linear pressure sensor array}

To test the correction of pressure measurements for inertial effects, experiments were carried out using a linear array of seven ultra-low range pressure sensors (Surrey Sensors Ltd. model ID7HP-250P-OEM). The sensors themselves are based on the Honeywell RSC-series architecture, which feature internal temperature compensation. The sensors used had a full-scale range of $\pm 250 \mathrm{~Pa}$, an uncertainty of less than $0.5 \%$ FS, a noise floor of less than $0.002 \%$ FS and a bandwidth of 1 $\mathrm{kHz}(9)$ The sensor array also features a high-sensitivity IMU (Bosch model BMI-160), which includes a 3-axis gyroscope having a full-scale range of $125 \%$ s and a 3 -axis accelerometer having a range of $19.6 \mathrm{~m} / \mathrm{s}^{2}$, both with an uncertainty of less than $\pm 0.0031 \%$ FS, a noise floor of less than $0.01 \%$ FS and a bandwidth of at least $1000 \mathrm{~Hz}$. The nominal relative positions of the sensors and the IMU are shown in fig. 2; the differences in the positions between the sensors and the IMU will allow more effective assessment of the applicability of eq. (4) as a generalized correction, as $\Delta x_{P} / L \gtrsim 40$. A second-order, centrebased numerical differentiation was used to obtain $\boldsymbol{\alpha}(t)$ from $\boldsymbol{\omega}(t)$.

For all tests, simultaneous pressure measurements were collected at $1 \mathrm{kHz}$ from all channels of the sensor array. Experiments were carried out in sufficiently short times that the effects of residual temperature sensitivity and drift on the measurements are negligible.

\section{B. Calibration for acceleration response}

Since $\rho, D$ and $h$ for the pressure sensor dies are unknown, the signal response of the sensors to acceleration needed first to be established emprically through calibration. To do this, the ports were all left open to atmosphere (so that $P^{*}=0$ ) and gravity was employed as a known $a_{P z}^{\prime *}$. First, pressures were measured while the sensors were nominally oriented so 


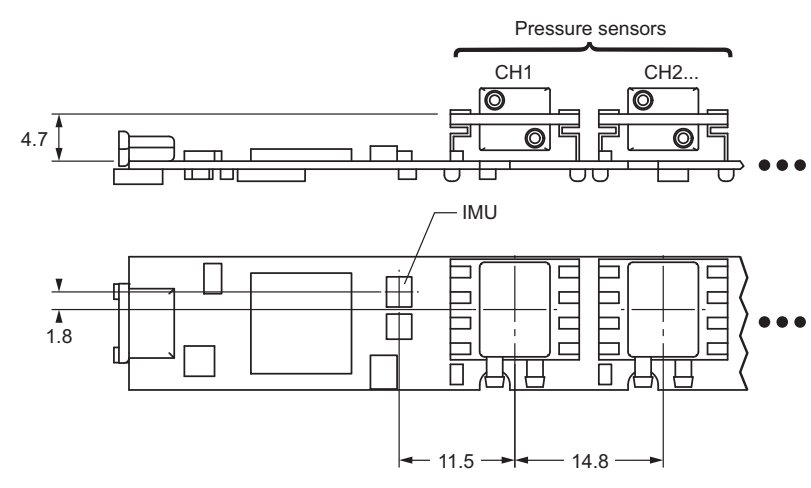

Fig. 2. Relative positions of IMU and pressure sensors on Surrey Sensors Ltd. ID7HP-250P-OEM. Only first two pressure channels shown for clarity. All dimensions are in $\mathrm{mm}$.
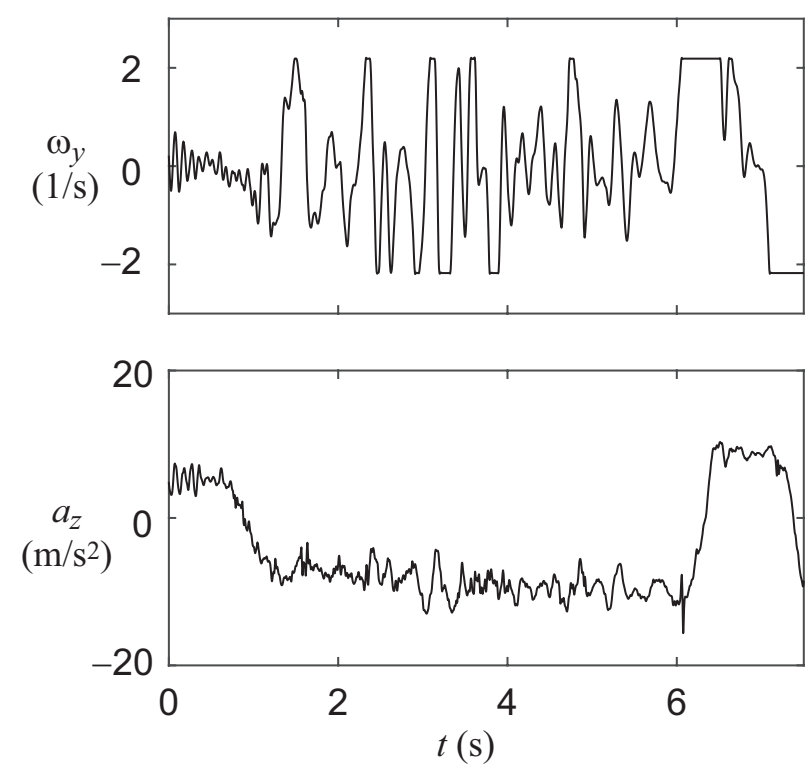

Fig. 3. Traces of $\omega_{y}$ and $a_{z}$ during arbitrary motion.

that gravity would apply the equivalent of a positive pressure $P_{g+}^{*}$; then, data were collected with the sensors oriented so that gravity would apply the equivalent of a negative pressure $P_{g-\text {. }}^{*}$. Since the effects of sensor nonlinearity and hysteresis were both negligible, the difference could be substituted into eq. (4) to yield the acceleration normalization factor,

$$
\frac{L^{3} \rho h}{D}=\frac{1}{2 g}\left(P_{g+}^{*}-P_{g-}^{*}\right)
$$

where $g$ is the acceleration due to gravity. The normalization factor is expected to be sensitive to the individual geometry and boundary conditions of each sensing element, and is therefore expected to vary significantly from unit-to-unit: for the seven sensor dies tested here, the unit-to-unit variation was as high as $\pm 23 \%$ from the mean.

\section{Testing in arbitrary, six-degree-of-freedom vibration}

With the sensors calibrated against acceleration, the proposed correction technique could be tested. The sensors were each hydraulically shorted by connecting the measurement
TABLE I

BASIC STATISTICAL CHARACTERISTICS OF RANDOM THREE-DIMENSIONAL MOTION APPLIED.

\begin{tabular}{|l||c|c|c|}
\hline Axis & Min & Max & St. Dev. \\
\hline$a_{x}\left(\mathrm{~m} / \mathrm{s}^{2}\right)$ & 10.32 & -10.38 & 2.39 \\
$a_{y}\left(\mathrm{~m} / \mathrm{s}^{2}\right)$ & 20.38 & -12.96 & 5.78 \\
$a_{z}\left(\mathrm{~m} / \mathrm{s}^{2}\right)$ & 10.52 & -16.89 & 5.15 \\
$\omega_{x}(1 / \mathrm{s})$ & $<-2.33$ & $>2.33$ & 0.77 \\
$\omega_{y}(1 / \mathrm{s})$ & $<-2.33$ & $>2.33$ & 0.94 \\
$\omega_{z}(1 / \mathrm{s})$ & $<-2.33$ & $>2.33$ & 0.93 \\
\hline
\end{tabular}

and reference pressure ports together with a short length of flexible hose, in order to ensure that they would necessarily be exposed to a constant, known pressure difference of $0 \mathrm{~Pa}$. The volume swept by the deforming membrane was estimated to be at least five orders of magnitude smaller than the volume of air inside the sensor enclosures, so any pressure losses due to fluid motion inside the shorting hoses were negligible. The ports could not be left open to atmosphere in this case, as the pressure differences generated by the motion of ambient air past the sensor ports during dynamic testing would dominate over momentum effects. Note that because of the high sensitivity of the sensors and the type of flexible fittings required by them, the application of highprecision prescribed nonzero or unsteady pressure differences for validation was not feasible.

The sensor array was then subjected to an arbitrary threedimensional motion in both translation and rotation. Figure 3 shows a sample of the components of acceleration and angular velocity obtained from the IMU during this motion, including the effect of gravity. The IMU had a maximum sensitivity to angular velocity of $\pm 2.33 \mathrm{~s}^{-1}$, and all three axes occasionally saturated for a brief period. The ranges of accelerations and angular velocities experienced are shown in Table I, along with their standard deviations.

Figure 4 shows the calibrated, dimensional pressure signals from the seven hydraulically-shorted differential pressure sensors during the same period of random motion shown in fig. 3. The signal noise is approximately $0.1 \mathrm{~Pa}$ in amplitude, so all other fluctuations here are caused by the momentum effects. The responses of the channels are different because of the effects of rotation, and the fluctuations have amplitudes as high as $\pm 1 \mathrm{~Pa}$ (equivalent to $0.4 \%$ FS). Since the fullscale uncertainty of the sensors is reported as $0.5 \% \mathrm{FS}$, the momentum effects clearly dominate.

With $\boldsymbol{\omega}(t), \boldsymbol{\alpha}(t)$, and $a_{o^{\prime} z}(t)$ from the IMU and the locations $\left(x_{P}, y_{P}, z_{P}\right)$ all known, the $z$-component of acceleration of each sensor could be evaluated using eq. (6) at each instant in time. The calibration from eq. (13) was then applied and the result substituted into eq. (4) to obtain the corrected values of $P^{*}$.

Figure 5 shows the corrected, dimensional pressures $P$ for all the sensors in the array, as functions of time. In all cases, the error in the pressure signals caused by membrane momentum effects have been significantly attenuated. Some residual nonzero measurements do remain (see especially channel 1 , for $6.5 \mathrm{~s} \lesssim t \lesssim 7.5 \mathrm{~s}$ ), but notably this also corresponds to times at which the angular velocity signals were saturated, leading to underpredicted corrections. The overall effect of the 


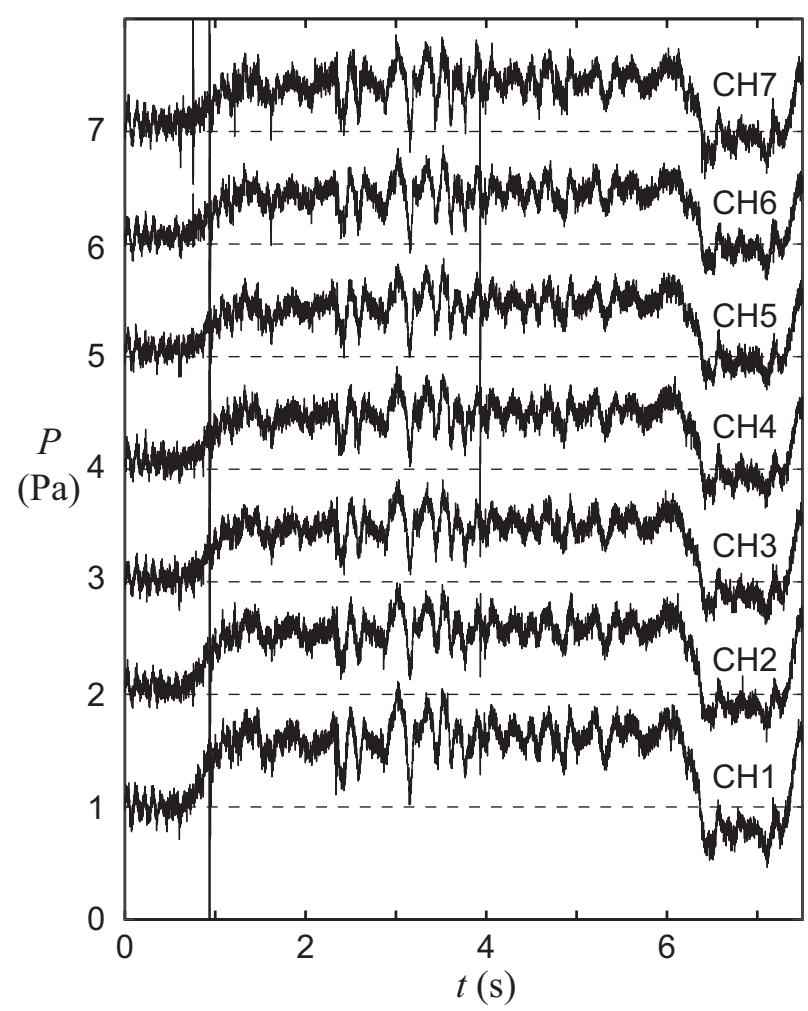

Fig. 4. Calibrated, dimensional readings of seven hydraulically-shorted differential pressure sensors during random motion. Note that channel signals have all been offset by $+1 \mathrm{~Pa}$ for clarity

TABLE II

PRESSURE SIGNAL QUALITY AFTER CORRECTING FOR MOMENTUM EFFECTS.

\begin{tabular}{|c||c|c|c|c|}
\hline \multicolumn{1}{|c||}{} & \multicolumn{2}{c|}{ Uncorrected $(\% \mathrm{FS})$} & \multicolumn{2}{c|}{ Corrected $(\% \mathrm{FS})$} \\
Sensor & $\Delta P$ & $\sqrt{\text { St.Dev. }}$ & $\Delta P$ & $\sqrt{\text { St.Dev. }}$ \\
\hline 1 & 0.662 & 0.199 & 0.412 & 0.139 \\
2 & 0.572 & 0.182 & 0.521 & 0.121 \\
3 & 0.631 & 0.176 & 0.441 & 0.118 \\
4 & 0.684 & 0.170 & 0.550 & 0.116 \\
5 & 0.621 & 0.167 & 0.506 & 0.120 \\
6 & 0.562 & 0.166 & 0.494 & 0.124 \\
7 & 0.846 & 0.171 & 0.800 & 0.126 \\
\hline
\end{tabular}

correction is tabulated in Table II. In all cases, the correction resulted in a reduction of the maximum pressure excursions, and the standard deviation was reduced by a factor of $\sim 2$.

\section{Significance of rotational terms}

To highlight the importance of including the effects of angular velocity and angular acceleration in the pressure correction, the ratio $\Psi$ between the angular correction terms and the linear correction terms in eq. (6) was defined, such that

$$
\Psi=\frac{\left(\omega_{x} \omega_{z}-\alpha_{y}\right) x_{P}+\left(\omega_{y} \omega_{z}+\alpha_{x}\right) y_{P}-\left(\omega_{x}^{2}+\omega_{y}^{2}\right) z_{P}}{a_{o^{\prime} z}}
$$

The magnitude of $\Psi(t)$ was then assessed for the same data as shown in fig. 4, and the time-mean $\overline{|\Psi(t)|}$ is tabulated in Table III for the seven sensors tested. The effects of angular motion contributed to between $10 \%$ and $37 \%$ of the correction,

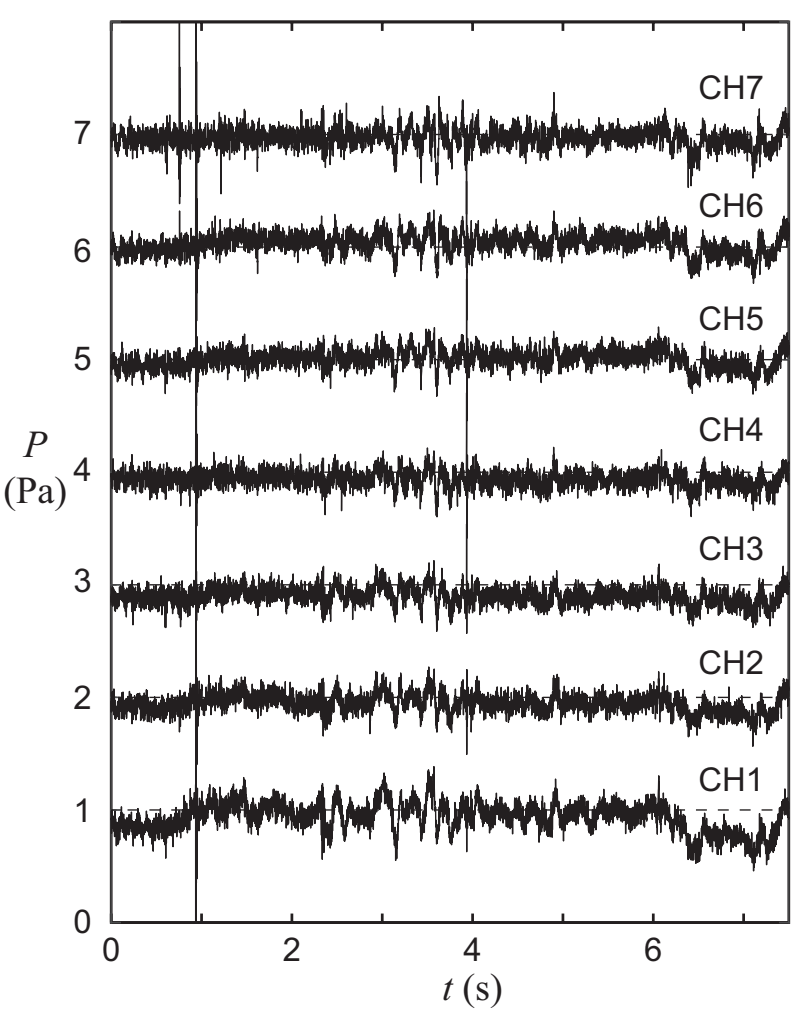

Fig. 5. Dimensional readings of the seven pressure sensors during random motion, corrected for momentum effects. Note that channel signals have all been offset by $+1 \mathrm{~Pa}$ for clarity

TABLE III

EFFECT OF ANGULAR TERMS ON THE PRESSURE SIGNAL QUALITY.

\begin{tabular}{|c|c|}
\hline Sensor & $|\Psi(t)|$ \\
\hline 1 & 0.096 \\
2 & 0.140 \\
3 & 0.184 \\
4 & 0.230 \\
5 & 0.275 \\
6 & 0.320 \\
7 & 0.366 \\
\hline
\end{tabular}

and increased with the distance between the pressure sensor and the IMU (as expected). Neglecting these terms (or using an inertial sensor insensitive to angular velocity) in this case would have resulted in a significant increase in uncertainty.

\section{Geometric UnCERTAinty}

Because the placement and orientation of both the IMU and pressure sensing dies are uncertain, a basic sensitivity analysis was carried out in order to establish the effect of geometric uncertainty on the pressure correction proposed.

\section{A. Translation}

The terms in eq. (6) representing the centripetal and tangential components of acceleration of the sensor dies will vary in relative magnitude depending on both the geometry of the PCB and the type of motion. In general, though, the placement error of components on a standard PCB (typically $\ll 1 \mathrm{~mm})$ will be small relative to the spacing $\left(x_{P}, y_{P}, z_{P}\right)$ 


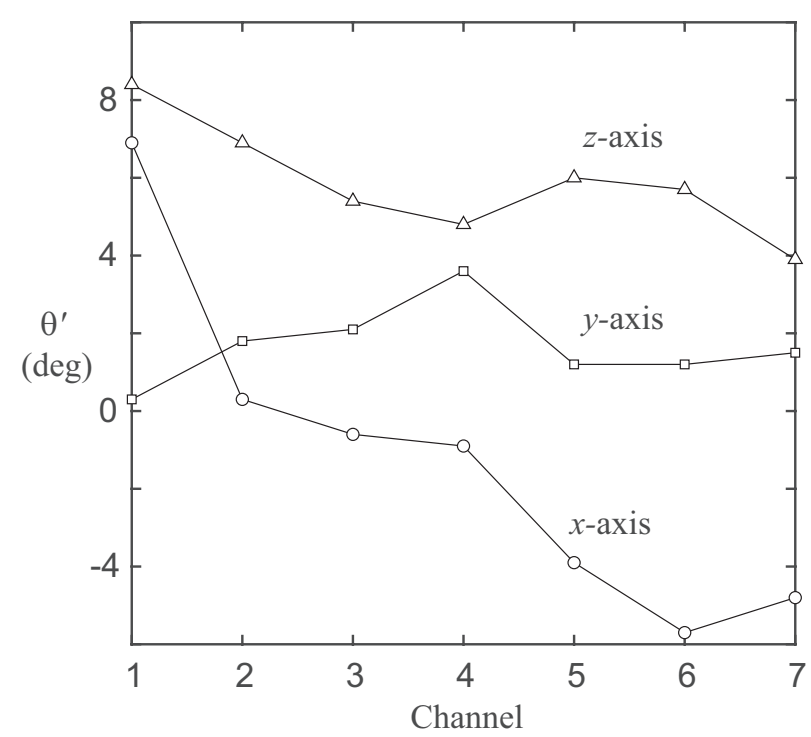

Fig. 6. Optimal rotational corrections for the seven pressure sensor dies.

between components, while $a_{o^{\prime} z}$ is invariant under translation. The effects of positional error are therefore expected to be insignificant. For the current arrangement of sensors, changes in the nominal values of $x_{P}, y_{P}$ and $z_{P}$ of as much as $10 \%$ had no observable effect on the corrected pressure signals.

\section{B. Rotation}

The relative orientation of the sensing elements, however, can be significant: this can result in components of any high in-plane accelerations (otherwise neglected) to act normal to the plane of the sensor die. For the case of surface-mounted IMUs, well-established procedures exist which use gravity to establish the orientation of their axes relative to the frame of the larger system; the same type of procedure cannot be applied to pressure sensors, as the magnitude of the signal response to gravity remains unknown.

To obtain an approximation of the orientation of the pressure sensors relative to the IMU, then, the sensors were again hydraulically shorted and subject to low-amplitude, random movement. Care was taken here not to saturate the IMU. In post-processing, A small but arbitrary rotation of the coordinate frame was applied to each sensor, and the accelerations resolved. A search process was carried out to identify the set of rotations which yielded the smallest standard deviation in the pressure signal, using the method described by Federov (10). The resulting 'optimal' rotations $\theta^{\prime}$ about each of the $x, y$ and $z$ axes are shown in fig. 6 . The trend (especially about the $x$ axis) suggests that there was likely a residual bend and/or twist in the PCB, but the rotations were all within $-6^{\circ} \lesssim \theta^{\prime} \lesssim 8^{\circ}$.

The effect of the angular re-alignment on the pressure correction was assessed by applying the 'optimal' rotations to eq. (5) and re-processing the same data set shown in fig. 4. The signal quality with and without the rotation is summarized in table IV. Overall, the effect of alignment correction was marginal, suggesting that electronic and other noise sources dominate over the uncertainty in sensor orientation.
TABLE IV

PRESSURE SIGNAL QUALITY AFTER RESOLVING ACCELERATIONS FOR DIE ROTATION.

\begin{tabular}{|c||c|c|c|c|}
\hline \multicolumn{1}{|c||}{} & \multicolumn{2}{c|}{ Unresolved (\%FS) } & \multicolumn{2}{c|}{ Resolved (\%FS) } \\
Sensor & $\Delta P$ & $\sqrt{\text { St.Dev. }}$ & $\Delta P$ & $\sqrt{\text { St.Dev. }}$ \\
\hline 1 & 0.412 & 0.139 & 0.403 & 0.136 \\
2 & 0.521 & 0.121 & 0.52 & 0.118 \\
3 & 0.441 & 0.118 & 0.432 & 0.115 \\
4 & 0.550 & 0.116 & 0.537 & 0.114 \\
5 & 0.506 & 0.120 & 0.506 & 0.119 \\
6 & 0.494 & 0.124 & 0.495 & 0.124 \\
7 & 0.800 & 0.126 & 0.798 & 0.126 \\
\hline
\end{tabular}

\section{DISCUSSION}

For membrane-type pressure sensors with very low fullscale ranges, the sensitivity to is so high that the effects of gravity alone can dominate the measurement uncertainty. Because the response of a thin membrane with locked boundary conditions to the application of body forces (and momentum pseudoforces) is similar to its response to applied surface pressure forces, the effect of acceleration may be treated as an additive 'pseudopressure' and eliminated in post-processing. This requires that the geometry is known and measurements of linear acceleration and angular velocity are available in the inertial frame of the pressure sensor die, but these requirements are easily met by including a typical MEMS IMU in the system design. Notably, the use of 'dummy' pressure sensors or linear accelerometers can result in unacceptable uncertainty if the spacing between the sensors is not sufficiently small. For distributed arrays of low-range pressure sensors, then, this approach of including the effects of rotation is necessarily required.

Once corrected for the effect of acceleration (which will include contributions both from motion and gravity), the full-scale uncertainty of the sensor can be reduced significantly. The correction algorithm is linear and time-stepping, and memory requirements are sufficiently small that realtime implementation in low-power microprocessors is feasible. The correction does require that each sensor be calibrated against acceleration, but this calibration does not require any applied pressure: the pressure ports need only be left open to atmosphere and data collected with the units in two fixed orientations.

The uncertainty in the positions of the sensors on the printed circuit (resulting from manufacturing or assembling variability) does not have a significant effect on the correction process and can therefore be neglected. The uncertainty in orientation is important and may depend on specific system geometry, but within typical automated PCB assembly tolerances, the effects were shown to nevertheless be negligible. The calibration of individual units to compensate for inaccuracy in assemblywhich can be tedious and add significantly to the computational resources required for data processing- is therefore largely unnecessary.

\section{CONCLUSIONS}

A simple technique for correcting signals from arrays of ultra-low range pressure sensors for the effects of acceleration 
has been demonstrated using a single inertial measurement unit. This correction was shown to reduce the full-scale uncertainty of the sensors by as much as $50 \%$ during random applied motion in translation and rotation. The technique necessarily requires unit-by-unit calibration against orientation, but only two calibration data points are needed. No high-precision calibration pressure source is required. For the case of a circuit board produced using typical automated manufacturing and soldering techniques, the effects of positional error in assembly were shown to have a negligible effect on the momentum correction.

\section{ACKNOWLEDGMENTS}

The authors would like to express their gratitude to Honeywell SIoT for their ongoing support.

\section{REFERENCES}

[1] Honeywell SIoT, “Trustability board mount pressure sensors, issue D," https://sensing.honeywell.com/honeywellsensing-trustability-rsc-series-data-sheet-32321348.pdf, 2020.

[2] D. M. Birch, "Uncertainty in pressure-based velocity probes," Surrey Sensors Ltd., Guildford, UK, Tech. Rep. TN-0614-2017 R1, 2017.

[3] S. Shaw-Ward, A. Titchmarsh, and D. M. Birch, "Calibration and use of $n$-hole velocity probes," AIAA Journal, vol. 53, no. 2, pp. 336-346, DoI 10.2514/1.J053130, 2015.

[4] S. Shaw-Ward, S. C. McParlin, P. Nathan, and D. M. Birch, "Optimal calibration of directional velocity probes," AIAA J., vol. 56, no. 7, pp. 2594-2603, 2017.

[5] B. Tian, Y. Zhao, and Z. Niu, "The study of acceleration effect for piezoresistive micro pressure sensor," Аpp. Mech \& Matl's, vol. 455, pp. 455-459, 2014.

[6] R. August, T. Maudie, and E. Thompson, "Acceleration sensitivity of micromachined pressure sensors," in Proceedings of the SPIE Conference on micromachined devices and components $V, 1999$, pp. 46-53.

[7] A. Kurtz, R. Ainsworth, S. Thorpe, and A. Ned, "Further work on acceleration insensitive semiconductor pressure sensors for high bandwidth measurements on rotating turbine blades," in NASA 2003 Propulsion Measurement Sensor Development Workshop, Huntsville, Alabama, 2003.

[8] S. Cai, W. Li, H. Zou, H. Bao, K. Zhang, J. Wang, Z. Song, and X. Li, "Design, fabrication and testing of a monolithically integrated tri-axis high-shock accelerometer in single (111)-silicon wafer," Micromachines, vol. 10, no. 227, pp. 1-11, 2019.

[9] Surrey Sensors Ltd., "Modular digital seven-hole probe system datasheet," https:/www.surreysensors.com/wpcontent/uploads/2016/09/DS-IDXHP-122019-1.1.pdf, 2020.

[10] V. V. Federov, Theory of Optimal Experiments. Academic Press, 1972.
Paul Nathan is the Technical Director and Head of Systems at Surrey Sensors Ltd., Guildford, UK

Rebecca Manning is a Design Engineer and Head of Naval \& Marine Applications at Surrey Sensors Ltd., Guildford, UK

David M. Birch is a Senior Lecturer in Aerospace Engineering at the University of Surrey, Guildford, UK, and Director of Research (Advanced Projects) at Surrey Sensors Ltd., Guildford, UK. He is also a Chartered Engineer. 